\title{
Análisis de la relevancia de la política pública para la atención de la salud mental en Costa Rica ${ }^{1}$
}

Daniel Martínez Esquivel ${ }^{2}$

\section{Institución: Universidad de Costa Rica}

\section{CÓMO CITAR}

\section{RESUMEN}

El presente ensayo pretende responder a la pregunta ¿cuál es la relevancia de las políticas públicas en la atención de la salud mental? Recientemente, se creó la Política Nacional de Salud Mental en Costa Rica aunque, a pesar de que significó un avance para el país, su implementación real y efectiva significa todo un reto. En la actualidad, la salud se concibe como un proceso histórico, afectado por diversas determinantes sociales que representan las posibles causas por las que las personas se enferman. Para atender dicha realidad, la Atención Primaria de Salud funge como una estrategia basada en la promoción de la salud, en la que la implementación de políticas públicas que dirijan las acciones del sector es una de sus áreas prioritarias. Desde esta perspectiva, la salud mental desempeña un papel trascendental en el bienestar del individuo y la comunidad. Se concluye que el desarrollo de una Política Nacional de Salud Mental representa un gran avance para Costa Rica; sin embargo, todos los profesionales de la salud deben conocerla y sensibilizar a sí y a la población en cuanto a atender desde la promoción de la salud mental y la prevención de la enfermedad mental.

Palabras clave: salud-mental, enfermería, políticas-públicas

\footnotetext{
${ }^{1}$ Fecha de recepción: 12 de diciembre 2013

Fecha de aceptación: 11 de febrero del 2014

${ }^{2}$ Enfermero. Estudiante de la Maestría Enfermería en Salud Mental y Psiquiatría de la Universidad de Costa Rica. Profesor de la Escuela de Enfermería de la Universidad de Costa Rica. Correo electrónico: dtinez@gmail.com
} 


\title{
Analysis of the relevance of public policy for mental health care in Costa Rica ${ }^{1}$
}

Daniel Martínez Esquivel ${ }^{2}$

\author{
Institution: University of Costa Rica
}

CITED

Martínez, D. (2014). Analysis of the relevance of public policy for mental health care in Costa Rica. Rev. Enfermería Actual en Costa Rica, 26, 18. Available: <http://www.revenf.ucr.ac.cr/saludmental.pdf> ISSN 1409-4568

\begin{abstract}
This essay seeks to answer the question what is the relevance of public policy in mental health care? Recently, the National Mental Health Policy was established in Costa Rica, but despite that was a step forward for the country, its real and effective implementation means a challenge. At present, health is seen as a historical process, affected by various social determinants that represent the possible reasons why people get sick. To address this reality, the Primary Health Care serve as one based on health promotion strategy in the implementation of public policies that direct the actions of the sector is one of its priority areas. From this perspective, mental health plays a major role in the welfare of the individual and the community. It is concluded that the development of a National Mental Health Policy represents a breakthrough for Costa Rica, but all health professionals should know and sensitize themselves and the public as to cater for the promotion of mental health and prevention of mental illness.
\end{abstract}

Keywords: mental-health, nursing, public-Policy

\footnotetext{
${ }^{1}$ Date of receipt: December 12, 2013

Date of acceptance: February 11, 2014

${ }^{2}$ Nurse. Master of Mental Health and Psychiatry Nursing student at the University of Costa Rica. Professor, School of Nursing at the University of Costa Rica.
} 


\section{INTRODUCCIÓN}

La evidencia científica actual facilita la comprensión de la salud desde una perspectiva distinta que se acompaña del paradigma social. Durante mucho tiempo, la salud fue comprendida a partir de una variable biológica, la cual se centró, específicamente, en la ausencia de enfermedad; sin embargo, el conocimiento del concepto ha evolucionado al igual que las transformaciones sociales en el mundo, cambio del que se concluye que la salud no es un proceso biológico, sino social. Al respecto, Organización Panamericana de la Salud (OPS, 2007), menciona que la salud se relaciona con variables sociales, económicas y políticas, ante todo, es un derecho humano innegable.

En cuanto a la salud y la enfermedad, es correcto afirmar que se producen social e históricamente de acuerdo con las condiciones de vida que, comparándolas entre sí, serán más saludables que otras (Benach y Muntaner, 2005); por consiguiente, la pobreza, el desempleo, el trabajo, la educación, el gradiente social, entre otros, son tan solo unas de las principales causas de enfermedad en la población. A pesar de tal descubrimiento, la inversión continúa centrándose en la curación y deja de lado otras estrategias de atención, fenómeno acerca del que Benach y Muntaner (2005) señalan que la mayor parte de los presupuestos de salud se invierten en hospitales; es decir, se trata la punta del problema y se descuida el verdadero origen del problema, omisión que ha generado que las tasas de incidencia y prevalencia sigan en aumento, dado que las causas existen en la sociedad.

Si bien el paradigma curativo está arraigado en algunos sistemas de salud mundiales, la Organización Mundial de la Salud (OMS, 2008) ha buscado provocar un cambio de enfoque en los servicios de salud, con el fin de promover estrategias que permitan a cada persona alcanzar el mayor grado de salud posible en sincronía con sus necesidades de salud desde la equidad y la solidaridad. Dicha estrategia se conoce como Atención Primaria de Salud (APS) y fue planteada en la Conferencia de Alma-Ata (1978); sin embargo, debido a las transformaciones sociales, la OMS ha replanteado su propuesta original al punto de renovarla considerando las demandas actuales.

La APS renovada segùn la OPS (2007) se fundamenta en los valores de derecho en cuanto al mayor nivel de salud posible, de equidad y de solidaridad, esenciales para establecer las prioridades de los sistemas de salud y evaluar si la atención satisface las necesidades de salud de la población. No obstante, rescatar esos valores no ha sido tarea fácil, razón por la que la OMS ha planteado un conjunto de reformas para articular una respuesta adecuada según los nuevos desafíos sanitarios:

Las reformas se centran en la cobertura universal para mejorar la equidad sanitaria, la prestación de servicios para construir sistemas de salud centrados en las personas, el liderazgo para que las autoridades sanitarias sean más confiables y las políticas públicas para promover y proteger la salud

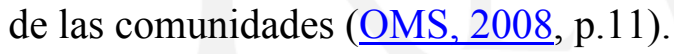

El presente ensayo se centra en la relevancia de las políticas públicas en la atención de la salud mental de las personas.

De acuerdo con lo presentado, se pretende responder a la siguiente interrogante, ¿Cuál es la relevancia de las políticas públicas en la atención de la salud mental? 


\section{DESARROLLO}

El análisis de este problema gira en torno de una perspectiva de Enfermería en Salud Mental, lo cual requiere comprender el marco de atención que se propone en la APS, modelo que rompe el enfoque curativo para favorecer la promoción de la salud. Según la OMS (1998), la promoción de la salud es el proceso en el que las personas pueden incrementar el control que tienen sobre su propia salud. Este concepto trae implícita la necesidad de que las personas sean quiénes se encarguen de su salud, aunque la promoción de la salud no abarca solo ese aspecto, sino que busca modificar las condiciones sociales, ambientales y políticas para mitigar su impacto en la salud, perspectiva que debe desarrollarse desde su estructura, de modo que implique acciones gubernamentales de cada Nación. En este sentido, la Carta de Ottawa (1986) establece como estrategia prioritaria de acción establecer políticas públicas favorables para la salud, idea que implica a los responsables de crear los programas políticos en todos los sectores y niveles, dado que cada inclusión debe ser inclusiva; es decir, procurar el bienestar general.

En la reforma impulsada por la OMS (2008), se promueve el establecimiento de políticas públicas que integren la intervención de salud pública y APS, medida que desea que se visualice la relevancia de las acciones intersectoriales, dado que se parte de que todo individuo es responsable por su salud; es decir, los profesionales de la salud no son los únicos encargados.

Las políticas públicas facilitan el desarrollo de la cohesión en el trabajo, puesto que desde estas se pauta un enfoque pensado en conjunto. Chiavenato (2011) menciona que una política es una regla establecida para gobernar funciones, guiar acciones y garantizar el cumplimiento de los objetivos trazados; aparte, se encargan de dotar de una identidad a las personas, debido a que consideran cada cosmovisión y el trabajo de las personas, de modo se satisface las necesidades de un individuo, comunidad o país desde una perspectiva contextual.

En Costa Rica, en distintas instituciones se han desarrollado políticas como la Política Nacional de Niñez y Adolescencia (2009-2021), la Política Nacional de Sexualidad (2010-2021), la Política Nacional de Seguridad Alimentaria y Nutricional (2011-2021), la Política Nacional para la Gestión Integral de Residuos (2010-2021), entre otras, que se ajustan al modelo de atención que se expone en este ensayo. Algunas de estas políticas provienen de otras, lo cual permite visualizar el proceso que se ha llevado a cabo. Al respecto, cabe mencionar que, hasta el año 2012, nunca se estableció una política dirigida a la salud mental.

El establecimiento de la Política Nacional de Salud Mental (2012-2021) significó un gran avance para el sistema de salud costarricense, atrasado en relación con el resto de naciones latinoamericanas, hecho lamentable si se ha comprendido la relevancia del Estado en la salud de la población, máxime que según Salud en las Américas 2007 (OPS, 2007), alrededor de un 73\% de los países americanos cuentan con políticas en salud mental.

Dentro de todo este marco de atención, la salud mental ocupa un lugar más que significativo, el cual define, en muchos casos, que las personas se enfermen o no. En relación con el tema, Malvárez (2009) comenta que la salud mental es la base para el bienestar y funcionamiento óptimo individual y comunitario, afirmación que significa que, si las personas gozan de un estado mental positivo, serán capaces de adaptarse a las condiciones sociales de una mejor manera, dado que mejora su capacidad de afrontamiento y fortalecen su salud. A pesar de tal cambio, la salud mental ha sido víctima de prejuicios que han llevado al estigma y la discriminación, debido a que quien padece una enfermedad mental está condenado a no tener oportunidades de desarrollo personal y social, 
al punto de que en la mayoría de los casos sus derechos son violentados, máxime que, como ,menciona Malvárez (2009), la salud mental y los derechos humanos constituyen una díada inseparable y fundamental para la promoción de la salud y por medio de la defensa de los derechos humanos se empodera a los individuos, las familias y las comunidades; la igualdad y la ausencia de la discriminación exige que se preste atención a los grupos más vulnerables.

De hecho, las principales causas de enfermedad mental son ajenas al sector salud y están influidas por factores sociales y económicos como por ejemplo la pobreza, la urbanización caótica, la carencia de vivienda, el desempleo, las condiciones laborales, la educación y la legislación penal (Rodríguez, 2009); por consiguiente, dichos factores, más que una intervención en salud, requieren una desde las políticas gubernamentales, acción que precisa sensibilizar y fortalecer toda la estructura estatal para que se trabaje sin obviar estas condiciones.

Si lo anterior se presenta a modo de, los resultados son interesantes: se estima que al menos un $25 \%$ de las personas padecen uno o más trastornos mentales o del comportamiento durante su vida (Rodríguez, Kohn y Levav, 2009), porcentaje que significa que 1 de cada 4 personas padecerá algún trastorno mental en algún momento de su vida, el cual le producirá sufrimiento psíquico que afectará su productividad y mantenimiento de la salud.

En Latinoamérica, la mayor parte de personas, del 25\% que padecen trastornos mentales, sufre de depresión o trastornos relacionados con el uso de alcohol y, en el tercer lugar, se ubica el trastorno obsesivo compulsivo. Lamentablemente, los países asignan menos del $2 \%$ del presupuesto de salud a la salud mental, aún cuando el 67\% está destinado a los hospitales psiquiátricos (OPS, 2012), monto que debería ser destinado a la promoción y la prevención a nivel comunitario. En Costa Rica, las estadísticas no son muy distintas: Díaz (2013), en un estudio de Unimer para el periódico La Nación, menciona que,

cerca de un $86 \%$ de los y las costarricenses dice sentirse satisfecho con su vida, sin embargo, un $77 \%$ dice sentirse estresado, mientras que un $60 \%$ dice haberse sentido deprimido y un $21 \%$ dice haber pensado en el suicidio. Por último, un $46 \%$ dice sentirse solo y un $34 \%$ contestó que, al menos una vez en su vida, se ha sentido sin esperanza.

A pesar de ser alarmantes, dichos datos no fueron comprendidos con la seriedad del caso, ya que socialmente la salud mental se vincula a la etiqueta "loco", cuando la verdad es que es un determinante de la salud que no desaparece de la cotidianeidad del individuo.

Otro dato relevante acerca de la salud mental aparece en el Atlas de la Salud Mental 2011 (OMS, 2011), en donde se menciona que la inversión diaria por persona en salud mental corresponde a 2 dólares estadounidenses, alrededor de 1000 colones, en países desarrollados; y en los países subdesarrollados se invierte cerca de 25 centavos estadounidenses, cifra que representa unos 125 colones diarios por persona y que es preocupante si se considera el costo de vida actual.

Los datos mencionados suscitan la necesidad de crear políticas públicas que para atender todas las causas de enfermedad; tal como se mencionó, el cambio debe implementarse desde la estructura para que el cambio se dé en todos los niveles. En cuanto a la salud mental, una política pública permite establecer valores, principios y 


\section{Revista Electrónica Enfermería Actual en costa Rica}

objetivos para mejorarla y reducir la carga de enfermedad en la población y define el nivel de prioridad de la salud mental en un gobierno y establece un modelo de actuación (Minoletti, Narváez, Sepúlveda y Funk, 2009).

Las políticas reflejan la forma de pensar de los gobiernos, así como sus estrategias para alcanzar el desarrollo social, a través de las que las personas conocen, de manera más clara, la línea de acción que deben seguir, por ende, es más asequible el cumplimiento de objetivos. En el 2012, el Ministerio de Salud de Costa Rica desarrolló la Política Nacional de Salud Mental, la primera de este tipo en la historia del país; para su elaboración, participaron sectores como medicina psiquiátrica, enfermería en salud mental, y psicología.

La Política Nacional de Salud Mental 2012-2021 (Ministerio de Salud, 2012) es un documento donde se definen principios, diversos enfoques y lineamientos para el desarrollo e implementación de un plan de acción de Salud Mental en Costa Rica. De acuerdo con el Ministerio de Salud (2012), la Política de Salud Mental integra un conjunto de valores, objetivos, lineamientos y estrategias, dirigidos a todos los sectores, con el propósito de desarrollar planes que contribuyan a mejorar la salud mental de la población en general. En esta política, la salud es concebida como producto de la sociedad (Ministerio de Salud, 2012), por tanto, la salud está condicionada por las distintas condiciones de vida de cada persona, aparte de que se entiende que la salud no es una situación biológica, exclusivamente, sino el resultado de un proceso histórico-social.

Los valores que justifican la política son equidad, universalidad, solidaridad, igualdad sustantiva, ética, calidad y calidez, y autonomía, cuyo propósito es establecer las prioridades nacionales de atención. También, la integran otros enfoques encargados de dirigir cada acción necesaria para que se cumplan los propósitos deseados; dichos enfoques versan acerca de derechos humanos, género, diversidad, integración, inclusión social, participación activa, desarrollo humano sostenible, evidencias científicas y buenas prácticas en Salud Mental (Ministerio de Salud, 2012). Alrededor de todo este gran marco conceptual es que se debe fundamentar la intervención en la salud mental, independientemente de la profesión que se ejerza.

Para la Enfermería en Salud Mental, la Política Nacional de Salud Mental es fundamental, puesto que apoya la perspectiva de Enfermería respecto del cuidado de la salud. De acuerdo con Neuman y Fawcett (2002), la Enfermería es la única profesión de la salud capaz de atender todas las variables que afectan a las personas en su entorno, debido a que su objetivo es valorar a la persona en relación con el ambiente para mantener el nivel más óptimo de salud del individuo, la familia y la comunidad a partir de la prevención. De igual forma, de acuerdo con Malvárez (2009), el objeto del cuidado en salud mental está orientado a establecer redes comunitarias centradas en promover, proteger, reestablecer y rehabilitar a las personas y grupos, para sustituir el modelo asilar de la psiquiatría. Desde este enfoque, la Enfermería debe dirigir su mirada hacia la comunidad porque es allí donde se encuentran los verdaderos problemas de salud y donde se pueden evitar; por tanto, la política apoya y fortalece este modelo.

Dado lo anterior, es necesario que todos los profesionales de enfermería conozcan sus planteamientos y conciencien al respecto, máxime que, como profesionales de un sistema de salud basado en APS, deben saber que las políticas y programas que estimulan la equidad son uno de los elementos de esta estrategia, razón por la que la OPS (2008) señala que todos los profesionales del sector salud deben conocer los marcos políticos y legales para eliminar las inequidades de salud de la comunidad: un gran compromiso pendiente. 
A pesar de que la política existe, el gran reto consiste en ejecutarla de manera efectiva y eficaz, puesto que el gran reto en la actualidad es la implementación real y efectiva de las políticas y planes nacionales en salud mental (Rodríguez, 2009), mas, si el objetivo no se logra, de nada servirá la implementación de nuevas políticas y todas las nuevas propuestas quedarán tan solo en la teoría.

La Política Nacional de Salud Mental representa el ideal de atención y servicios que se deben prestar en el país para atender la salud mental. Sin embargo, de acuerdo con Sisy Castillo, viceministra de salud mental, sin presupuesto ni un órgano que vele por su cumplimiento, la implementación de la política está cuesta arriba (Díaz, 2013), afirmación de la que es posible observar una ambivalencia en el Estado que restringe los alcances de la política. De momento, es tarea de cada profesional en Enfermería conocerla e implementarla en su lugar de trabajo.

El debate queda abierto, ahora es cuestión de impulsar la Política Nacional de Salud Mental para sensibilizar a la población en general, encargada de crear y empoderarse de las nuevas propuestas para mirar la salud, las cuales se alejan del paradigma curativo y dan la bienvenida al paradigma de promoción de la salud, una medida que busca mantener la salud de las personas en favor de su desarrollo personal.

\section{CONCLUSIÓN}

El desarrollo de una Política Nacional de Salud Mental representa un gran avance para Costa Rica; sin embargo, todos los profesionales de la salud deben conocerla y sensibilizar a sí y a la población en cuanto a atender desde la promoción de la salud mental y la prevención de la enfermedad mental.

La Enfermería en Salud Mental es líder en este cambio de paradigma ya que atiende a todos los determinantes de la salud que afectan a individuos y comunidades, porque cuenta con el conocimiento y las habilidades necesarias para generar planes, programas y proyectos de acción que respondan a todas las necesidades de salud mental.

\section{REFERENCIAS BIBLIOGRAFÍCAS}

Benach, J. y Muntaner, C. (2005). Aprender a mirar la salud, ¿cómo la desigualdad social daña nuestra salud? Maracay, Venezuela: IAESP "Dr. Arnoldo Gabaldon”.

Chiavenato, I. (2011). Administración de recursos humanos. El capital humano de las organizaciones. Novena edición. México D.F.: McGraw Hill.

Díaz, L. (2013, 25 agosto). Males mentales en Costa Rica: detrás del 'pura vida' hay un tico con estrés y depresión. La Nación.

Díaz, L. (2013, 25 agosto). Política de Salud Mental está solo en el papel. La Nación.

Gutiérrez, A., Meza, M. (1997/1998). Derechos Humanos de una población olvidada: personas con discapacidad mental. Rev. Latinoam. Der. Méd. Medic. Leg. 2(2) / 3(1): 65-69. 


\section{Revista Electrónica Enfermería Actual en costa Rica}

Malvárez, S. Promoción de la salud mental. En Rodríguez, J. (2009). Salud Mental en la Comunidad. Segunda Edición. Washington D.C.: OPS.

Ministerio de Salud. (2012). Política Nacional de Salud Mental 2012-2021. San José: Ministerio de Salud.

Minoletti, A., Narváez, P., Sepúlveda, R. y Funk, M. Desarrollo de políticas y planes de salud mental comunitaria.

Rodríguez, J. (2009). Salud Mental en la Comunidad. Segunda Edición. Washington D.C.: OPS.

Neuman, B. y Fawcett, J. (2002). The Neuman Systems Model.Fourth Edition. New Jersey: Prentice Hall.

OMS (2011). Atlas de la Salud Mental 2011. Ginebra: OMS.

OMS (2008). La atención primaria de salud. Más necesaria que nunca. Ginebra: OMS.

OMS (1998). Promoción de la Salud. Glosario. Ginebra: OMS.

OMS (1986). Carta de Ottawa para la promoción de la salud. Ottawa: OMS.

OMS/OPS y UNICEF (1978). Declaración de Alma-Ata. Conferencia Internacional sobre Atención Primaria de Salud. URSS: OMS.

OPS (2012). Salud en las Américas 2012. Panorama regional y perfiles de país. Publicación científica y técnica No. 636. Washington D.C.: OPS.

OPS (2008). Estrategias para el desarrollo de los equipos de APS. Washington D.C.: OPS.

OPS (2007). La Renovación de la Atención Primaria de Salud en las Américas. Washington D.C.: OPS.

OPS (2007). Salud en las Américas 2007. Publicación científica y técnica No. 622, Vol. 1. Washington D.C.: OPS.

Rodríguez, J. (2009). Salud Mental en la Comunidad. Segunda Edición. Washington D.C.: OPS.

Rodríguez, J., Kohn, R. y Levav, I. (2009) Epidemiología de los trastornos mentales en América Latina y el Caribe. En Rodríguez, J. Salud Mental en la Comunidad. Segunda Edición. Washington D.C.: OPS.

Wilkinson, R. y Marmot, M. (2003). Determinantes sociales de la salud: los hechos irrefutables. Ginebra: OMS. 